\title{
A valuation model of credit-rating linked coupon bond based on a structural model
}

\author{
K. Yahagi \& K. Miyazaki \\ The University of Electro-Communications, Japan
}

\begin{abstract}
A credit-linked coupon bond pays a coupon associated with its credit rating at the time of the coupon payment date, rather than an amount equal to the initially fixed coupon. The only existing corporate bond valuation model for creditrating-triggered products was formulated by Jarrow et al. However, this model does not incorporate the fact that increases in the coupon payment resulting from downgrades may cause a further deterioration of credit ratings and of the likelihood that the company will be able to make future coupon payments. In this paper, we present a credit-linked coupon bond valuation model that considers this issue. Using a structural approach, we extend the classical model of Merton by introducing a threshold value corresponding to each credit rating, and a volatility of the company value process that depends on its credit rating. Given these extensions, our model is more flexible than the JLT model, and we are clearly able to capture the above effect via numerical simulations. Furthermore, from the perspective of practical implications, the JLT model tends to value credit-linked coupon bonds more cheaply than does our model when the initial credit rating is high, while the reverse is true for a low initial credit rating. Keywords: risk management, derivative pricing, credit risk.
\end{abstract}

\section{Introduction}

The formulation and use of corporate bond valuation models dates from the work of Merton [5]. In the Merton model, the default of a bond is defined as a state in which the corporate value falls below the face amount of the bond, and in which the corporate value process follows a geometric Brownian motion. As a result of these assumptions, the Merton model may easily be used in conjunction with the Black-Scholes formula to value corporate bonds. Using valuation frameworks of this kind is typically characterised as following a "structural approach," and 
many extensions of the Merton model have been derived. Another avenue for corporate bond valuation is relatively new and is known as the "reduced form approach." The latter approach assumes that the time to default may be modelled as a hazard rate. Famous and representative reduced form models include those of Jarrow and Turnbull [4] (the JT model), Jarrow et al. [3] (the JLT model), and Duffie and Singleton [2]. Among these structural and reduced form models, only the JLT model explicitly uses a rating transition matrix in modelling the time to default.

Given such preceding research on the valuation of the corporate bond, the JLT model at first glance appears the most suitable for the valuation of creditrating-triggered bonds, such as the credit-rating-linked coupon bond. However, in order to incorporate the idea that the increased coupon payment due to downgrading deteriorates the potential for future coupon and notional payments, the impact of increased coupon payments on the balance sheet of the company must be considered, in addition to the credit-rating transition itself. In this paper, for the purpose of valuing credit-rating-linked coupon bonds, we further develop the ideas presented by Bhanot [1] by considering an analogue of the JLT model in a structural context.

The remainder of the paper is organised as follows. The next section briefly reviews the Merton and JLT models, and presents the motivation for our research. Section 3 proposes our valuation model and its means of calibration. Section 4 examines various features of the model using numerical examples. The final section summarises and concludes.

\section{Prior research and the motivation for our model}

\subsection{Merton model}

The Merton model assumes that the value of the company follows a next geometric Brownian motion:

$$
\frac{d V_{t}}{V_{t}}=\mu d t+\sigma d W_{t}
$$

where $\mu, \sigma$, and $W_{i}$ are, respectively, the drift and volatility of the corporate value process and a standard Brownian motion under the usual statistical measure.

In order to value a corporate bond, the Merton model first transforms process (1) into one under a risk-neutral probability measure, such as process (2) below:

$$
\frac{d V_{t}}{V_{t}}=r d t+\sigma d \widetilde{W}_{t}
$$

where $r, \sigma$, and $W_{t}$ are, respectively, the risk-free short rate, the volatility of the corporate value process, and a standard Brownian motion under the usual riskneutral measure.

The model then computes the risk-neutral expectation of the payoff expressing the corporate bond value $\min \left(V_{r}, B\right)$, where $B$ denotes the face amount of the bond. Finally, the model discounts this expectation back to its 
present value. Therefore, the model makes convenient use of the Black-Scholes formula.

\subsection{The JT and JLT models}

\subsubsection{The JT model}

Under an appropriate probability space and the assumption that the risk-free interest rate process and the default time process are independent, the JT model provides the value $(F(t, T))$ of the $T$-maturity discount corporate bond at time $t$ as given by equation (3):

$$
F(t, T)=p(t, T)\left(\delta+(1-\delta) \widetilde{Q}_{t}\left(\tau^{*}>T\right)\right),
$$

where $\delta$ is the recovery rate, $p(t, T)$ is the price of the $T$-maturity risk-free discount bond at time $t$, and $\widetilde{Q}_{t}\left(\tau^{*}>T\right)$ is the probability under the risk-neutral probability measure that the default happens after the maturity of the bond.

\subsubsection{The JLT model}

The JLT model first describes the credit rating of a company using the state space $S=\{1, \ldots, k\}$. The first state indicates the highest credit rating (AAA), while the second state corresponds to the second-highest credit rating (AA), and so on. The final state $k$ indicates default. The model initially adopts matrix (4) as the credit-rating transition probability matrix for a given point in time. In particular, the empirical credit-rating transition probability matrix is given by

$$
Q=\left[\begin{array}{cccc}
q_{1,1} & q_{1,2} & \cdots & q_{1, k} \\
q_{2,1} & q_{2,2} & \cdots & q_{2, k} \\
\vdots & \vdots & \ddots & \vdots \\
q_{k-1,1} & q_{k-1,2} & \cdots & q_{k-1, k} \\
0 & 0 & \cdots & 1
\end{array}\right] .
$$

where $q_{i, j}$ is the probability that the credit rating of the company changes from $i$ to $j$, and where, for all $i, j, q_{i, j} \geq 0$ and $q_{i, i}(t, t+1) \equiv 1-\sum_{\substack{i=i \\ j \neq i}}^{k} q_{i, j}(t, t+1)$. Moreover, the $n$-period transition probability matrix is then computed as $Q_{0, n}=Q^{n}$.

Under the usual assumptions that the market is complete and that the arbitrage-free condition is satisfied, the JLT model then introduces the transition probability matrix from time $t$ to time $t+1$ under a risk-neutral measure:

$$
\widetilde{Q}_{t, t+1}=\left[\widetilde{q}_{i, j}(t, t+1)\right] .
$$

To retain its Markov character, the JLT model restricts the risk-neutral probability $\tilde{q}_{i, j}(t, t+1)$ to

$$
\widetilde{q}_{i, j}(t, t+1)=\pi_{i}(t) q_{i, j}
$$

for all $i, j, i \neq j$, where $\pi_{i}(t)$ is the risk premium. The matrix form of equation (6) may be written as

$$
\widetilde{Q}_{t, t+1}-I=\Pi(t)[Q-I]
$$


where $I$ is a $k \times k$ unit matrix $\Pi(t)=\operatorname{diag}\left(\pi_{1}(t), \ldots, \pi_{k-1}(t), 1\right)$, for all $i, j$, $\pi_{i}(t)>0$. Furthermore, $\widetilde{q}_{i, j}(0, n)$ is defined as the probability that the credit rating of the company jumps from credit rating $i$ to credit rating $j$ over $n$ periods, and this probability is expressed as the $(i, j)$-th entry on the left side of equation (8).

$$
\widetilde{Q}_{0, n}=\widetilde{Q}_{0,1} \widetilde{Q}_{1,2} \cdots \widetilde{Q}_{n-1, n} .
$$

Under the risk-neutral probability measure, the JLT model provides the probability $\widetilde{Q}_{t}^{i}\left(\tau^{*}>T\right)$ that the a company with the $i$-th credit rating at time $t$ does not default until the maturity $T$ of the bond as

$$
\tilde{Q}_{t}^{i}\left(\tau^{*}>T\right)=\sum_{j \neq K} \tilde{q}_{i, j}(t, T)=1-\tilde{q}_{i, k}(t, T),
$$

where $\tau^{*}=\inf \left\{s \geq t: \eta_{s}=k\right\}$.

Using equation (10), the JLT model then evaluates the $T$-maturity, $i$-th credit rating discount corporate bond at time $t, F^{i}(t, T)$, simply by substituting $\widetilde{Q}_{t}^{i}\left(\tau^{*}>T\right)$ in place of $\widetilde{Q}_{t}\left(\tau^{*}>T\right)$ in valuation formula (3) of the JT model.

$$
F^{i}(t, T)=p(t, T)\left(\delta+(1-\delta) \widetilde{Q}_{t}^{i}\left(\tau^{*}>T\right)\right) .
$$

\subsection{Characteristic features of the Merton and JLT models, and the motivation for our model}

\subsubsection{The Merton model}

Strength:

Since it integrates a default based on the structure of the balance sheet of the company, the model easily incorporates the financial impact of credit-rating changes on the balance sheet.

Weaknesses:

1. The model does not explicitly describe credit ratings and, therefore, is not suitable for valuing credit-rating-triggered products.

2. With the exceptions of the risk-free interest rate $r$ and the maturity $T$ of the bond, the model has only three fundamental parameters, namely the volatility of the corporate value process $\sigma$, the initial corporate value $V_{0}$, and the face amount of the corporate bond $B$. Therefore, the model has too few parameters to fit the market credit spreads of all maturities flexibly.

3 . In this regard, the volatility $\sigma$ of the company value process does not depend on its credit rating and is constant across all credit states.

4. In the course of valuing a coupon bond, the model must determine whether the bond was in default at any coupon payment date, and this procedure is very timeconsuming.

5. The model cannot incorporate the term structure of risk-free interest rates.

\subsubsection{The JLT model}

Strengths:

1. The model is based on credit ratings and is therefore suitable for valuing credit-rating-triggered products. 
2. The model incorporates a credit risk premium $\pi_{i}(t)$ that depends both on the time $t$ and the credit rating $i$ provided in the risk-neutral credit-rating transition probability matrix $\widetilde{Q}$. Therefore, the model is flexible enough to fit market credit spreads for all maturities.

3 . In this regard, not only the risk premium $\pi_{i}(t)$, but also the empirical creditrating transition probability $q_{i, j}$ in the matrix $Q$, depend by definition on the credit rating.

4. The model easily values coupon bonds.

5. The model is able to incorporate the term structure of risk-free interest rates.

Weakness:

Since it models a default using a credit-rating transition probability matrix, the model does not incorporate the structure of the balance sheet of the company. For this reason, it does not consider the financial impact of the credit rating on the balance sheet.

In light of these characteristics, we propose a valuation model for the creditrating-linked coupon bond that incorporates the impact of increased coupon payments on the potential of the firm to pay future coupons and to make face value payments. Our modelling approach is structural, although we recognise that structural models are in several respects weak in comparison to the JLT model. In short, we attempt to incorporate the benefits of the JLT model into an analogous structural model.

\section{Our model and its calibration}

\subsection{Our model}

Before introducing our model, we describe the correction of several weaknesses of the Merton model:

Weakness 1

As an analogue of the credit-rating state space $S=(1, \ldots, k)$ in the JLT model, we introduced $k-1$ threshold values, $V^{*(i)}, i=1, \cdots, k-1$. The $k-1$-th threshold value $V^{*(k-1)}$ is simply the coupon value $c^{(k-1)}$ of the bond at the coupon payment date and the face amount $B+c^{(k-1)}$ of the bond at Maturity.

Weaknesses 2 and 3

Instead of the common volatility of the corporate value process $\sigma$, we introduced the credit-rating-dependent volatilities $\sigma^{*(i)}$, for $i=1, \cdots k-1$. In the case of $i=k$, no volatility exists, because the company defaults in that state. The volatility $\sigma^{*(i)}$ essentially corresponds to the empirical credit-rating transition probability matrix $Q$ in the JLT model. We also introduced a credit-ratingdependent initial corporate value $V_{0}^{i}$, for $i=1, \cdots k-1$, to increase the flexibility of the model.

Weakness 4

Since we adopted a Monte Carlo simulation method for the purpose of valuation, the analysis required very little time. 
Our model:

Based on these revisions, the risk-neutral company value process in our model may be described as in equations (11) and (12) below.

At any time except that of the coupon payment,

$$
\begin{array}{cl}
d V_{t}^{i}=r V_{t}^{i} d t+\sigma^{*(1)} V_{t}^{i} d W_{t}, & : V_{t}^{i}>V^{*(1)} \\
d V_{t}^{i}=r V_{t}^{i} d t+\sigma^{*(j)} V_{t}^{i} d W_{t} . & : V^{*(j-1)}>V_{t}^{i}>V^{*(j)}
\end{array}
$$

In addition, at the coupon payment time $t_{l}$,

$$
V_{t_{l}}^{i}=V_{t_{l^{-}}}^{i}-c^{(j)} . \quad: V^{*(j-1)}>V_{t_{l^{-}}}^{i}>V^{*(j)}
$$

where, $V_{t^{-}}^{i}$ is the just-before- $t_{l}$ value of the corporate bond with initial credit rating $i$, and where $c^{(j)}$ is the coupon of a bond with the $j$-th credit rating at the date of issue.

Valuation procedure based on a Monte Carlo simulation:

Step1:Simulate the sample path of the corporate value process given by equations (11) and (12), starting with the initial corporate value.

Step2 :Compute the cash flow (coupon + face amount) for each sample path.

Step 3 :Invest the cash flow calculated in Step 2 in the risk-free asset for the maturity $T$ of the corporate bond. Take the risk-neutral expectation of the invested cash flow at time $T$, and discount it backwards to its present value.

\subsection{Calibration of our model}

\subsubsection{Parameters in our model}

Exogenous parameters:

The exogenous parameters include the credit-rating-dependent company value volatilities $\sigma^{*(i)}$, for $i=1, \cdots k-1$, as well as the coupon and face amounts of the bond, $c^{(j)}$ and $B$. As mentioned above, these values correspond to the empirical credit-rating transitional probability matrix $Q$ in the JLT model.

Parameters to be estimated:

The parameters to be estimated included the credit-rating-dependent initial corporate values $V_{0}^{i}$, for $i=1, \cdots k-1$, and $k-2$ threshold values, such as state $V^{*}(i)$, for $i=1, \cdots k-2$, except the default state $V^{*(k-1)}$ and the total number of parameters was $2 k-3$. To facilitate the calibration of the model, we restricted the $k-1$ threshold values $V^{*(i)}$, for $i=1, \cdots k-2$, by $V^{*(i)}=\left(V_{0}^{i}+V_{0}^{i+1}\right) / 2$, for $i=1, \cdots, k-2$, by $V^{*(k-1)}=c^{(k-1)}$ at the coupon payment date, and by $V^{*(k-1)}=B+c^{(k-1)}$ at maturity. Therefore, the total number of parameters to be estimated was simply $k-1$.

The $k-1$ initial company values $V_{0}^{i}$, for $i=1, \cdots k-1$, in our model correspond to the risk premium $\pi_{i}(t)$ in the JLT model. We allowed the initial company values $V_{0}^{i}$, for $i=1, \cdots k-1$, to depend on the maturity $T$ of the 
corporate bond. Under this allowance, the number of parameters $\pi_{i}(t)$ in the JLT model (discrete version) matches that of the parameters $V_{0}^{i}$ in our model.

\subsubsection{Calibration}

Three remarks regarding the model calibration are in order. First, we allowed the initial company values $V_{0}^{i}$ to depend on the maturity $T$ of the corporate bond. Therefore, the estimated values of $V_{0}^{i}$ could differ by maturity. Second, for each maturity $T$, we tried to estimate the $k-1$ initial company values by fitting the $k-1$ model credit spreads to the market credit spreads by numerically solving $k-1$ equations. Finally, we assumed that the coupon bonds observed in the market were par bonds, and that their coupons were the same as their yields.

\section{Numerical experiments}

Specification of the credit-rating-linked coupon bond, and valuation methods in numerical experiments:

Each credit-rating linked coupon bond was assumed to behave as follows. If the bond bore the same credit rating that it had on issuance, then it paid at each coupon date the amount of the corresponding coupon initially specified. If the bond was in default at the coupon payment date, the corporate value at that time was paid at the maturity $T$ of the bond.

In several numerical experiments, we compared the various bond values derived from the three different valuation models: (1) the JLT model, in which, at the coupon payment date, the coupon corresponding to the credit rating was paid, as mentioned above; (2) Model A (our model); and (3) Model B, which was essentially the same as our model, except that the fall in company value resulting from coupon payments remained at the initial coupon amount, although the company paid the coupon corresponding its credit rating at the coupon payment date. In other words, we adopted a model that was economically incorrect as a reference point from which to evaluate the other models.

Data and the setting of external parameters:

We adopted six possible credit ratings: AAA, AA, A, BBB, BB, and D. Therefore, $k=6$. The bond maturity was five years, and the term structure of the risk-free interest rate was flat. The face amount of each bond was 70 yen, and the coupon of the bond with each credit rating was the same as its yield.

Table 1: The credit spreads.

Table 2: The volatilities.

\begin{tabular}{cccccc}
\hline Rating & AAA & AA & A & BBB & BB \\
\hline Steep & $5 \%$ & $10 \%$ & $20 \%$ & $25 \%$ & $35 \%$ \\
Flat & $20 \%$ & $20 \%$ & $20 \%$ & $20 \%$ & $20 \%$ \\
\hline
\end{tabular}

\begin{tabular}{cccccc}
\hline Rating & AAA & AA & A & BBB & BB \\
\hline Steep & $0.18 \%$ & $0.44 \%$ & $0.92 \%$ & $1.85 \%$ & $4.69 \%$ \\
Flat & $0.16 \%$ & $0.26 \%$ & $0.46 \%$ & $1.12 \%$ & $2.05 \%$ \\
\hline
\end{tabular}

We adopted the average empirical credit-rating transition probability matrix $Q$ in the JLT model that was announced by R\&I (a Japanese rating agency) between 1994 and 2004. In this derivation, we lumped together all of the transition probabilities for credit ratings below $\mathrm{BB}$, with the exception of the default state; these were given the corresponding credit-rating label "BB." 
Moreover, in estimating the risk premium $\Pi(t)$, we used the estimation technique adopted by JLT (1997).

Table 3: $\quad$ The cases of numerical experiments.

\begin{tabular}{ccc}
\hline The Cases of Credit Spread(Flat): & Volatilities(Flat) & Volatilities(Steep) \\
\hline Risk-free interest rate(1.21\%) & Case 1 & Case2 \\
Risk-free interest rate(3.21\%) & Case3 & Case4 \\
\hline \hline The Cases of Credit Spread(Steep): & Volatilities(Flat) & Volatilities(Steep) \\
\hline Risk-free interest rate(1.21\%) & Case5 & Case6 \\
Risk-free interest rate(3.21\%) & Case7 & Case8 \\
\hline
\end{tabular}

For both the volatility of the company value process and the credit spread of the bond corresponding to each credit rating, we allowed two different settings, and these are listed in Tables 1 and 2, respectively. In addition, we set the riskfree interest rate alternatively at $1.21 \%$ and $3.21 \%$. Therefore, in total, we performed eight numerical experiments (Cases 1 through 8), the results of which are summarised in Table 3.

The results of the numerical experiments, and their implications:

The eight valuations, corresponding to Cases 1 through 8 , of the credit-ratinglinked coupon bond for each of the three valuation models are provided in Figures 1 through 8 , respectively.

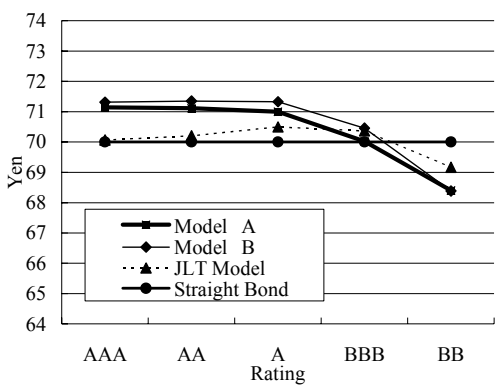

Figure 1: $\quad$ The results of Case 1.

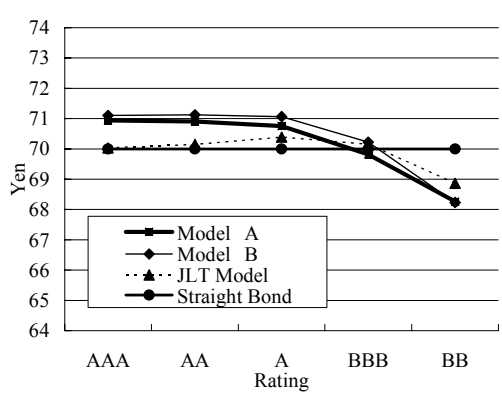

Figure 3: $\quad$ The results of Case 3.

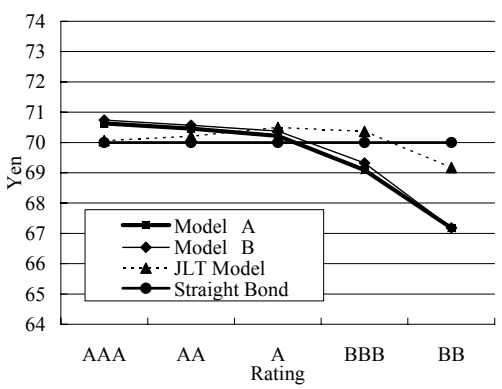

Figure 2: $\quad$ The results of Case 2.

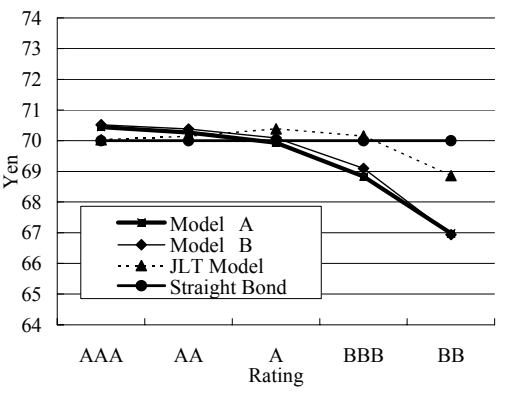

Figure 4: $\quad$ The results of Case 4. 


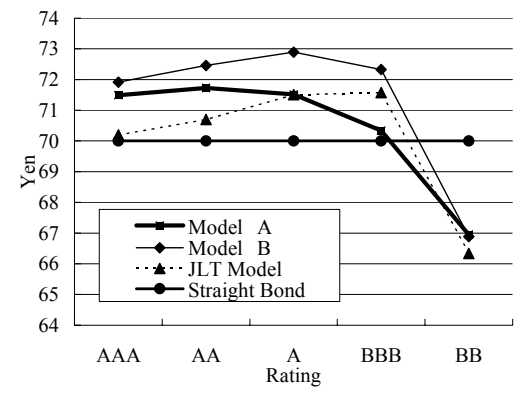

Figure 5: $\quad$ The results of Case 5.

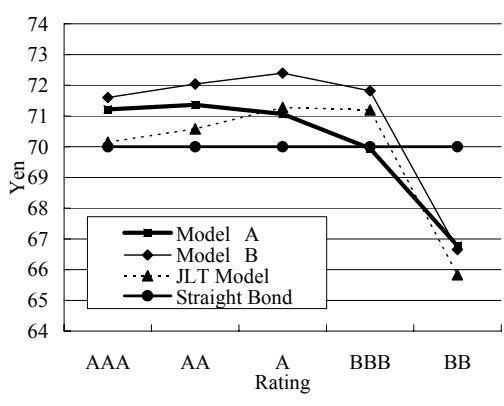

Figure 7: The results of Case 7.

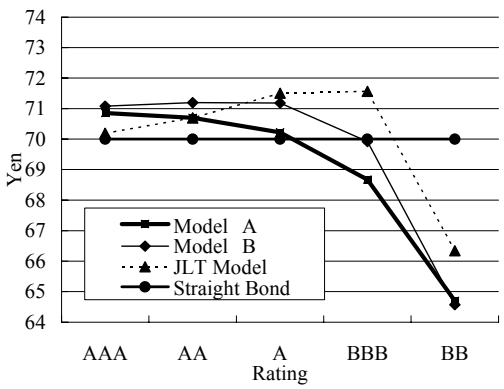

Figure 6: The results of Case 6.

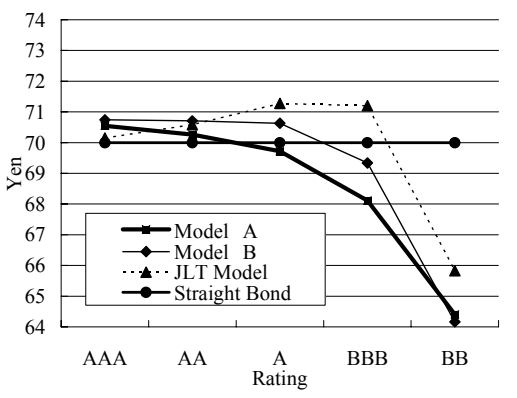

Figure 8: The results of Case 8 .

(1) Overview of the results

(a) All three models valued the credit-rating-linked coupon bond above the straight bond when the credit rating of the bond was relatively high (AAA, AA, $\mathrm{A}$ ), while the opposite was true when the credit rating of the bond was relatively low (BBB, BB).

(b) The value of the credit-rating-linked coupon bond derived from the JLT model tended to be lower than those derived from Model A and Model B under a relatively high initial credit rating (AAA, AA, A); the reverse was true under a relatively low initial credit rating.

The first result was obtained because, under a higher initial credit rating, the effect of the coupon increase resulting from a downgrade swamped the resulting decrease in the potential of the company to make future coupon payments. Under a low initial credit rating, the situation was reversed. The second result was obtained because the coupon payment amount did not affect the credit-rating transition probability in the JLT model, while the increasing coupon amount increased the default probability, and the magnitude of this effect was larger under a low credit rating than under a high credit rating.

(2) The influence of the credit spread (comparison of Case $1 \&$ Case 4 and Case 5 \& Case 8).

The first result (1) appeared more salient for a large, steep credit-spread curve than for one that was small and flat. The reason underlying the first result in (1) also explains this observation. 
(3) The influence of the volatility of the company value process (comparison of Cases 5 and 6)

(a) In both Models A and B, and for all credit ratings, the value of the creditrating-linked coupon bond tended to be higher under a flat volatility structure (20\% in all cases) than under a steep volatility structure $(5,10,20,25$, and $35 \%$, respectively, from the highest credit rating to the lowest).

(b) The valuation derived using Model B deviated from that of Model A to a greater extent under the flat volatility structure than under the steep one.

The first result may be explained as stemming from reason (1) above. The deviation of the value derived from Model B from that derived from Model A resulted from both the credit-rating probability and the difference between the initially set constant coupon and the credit-rating-linked coupon. For Cases 5 and 6 , the latter impact was the same, but the former was larger under flat volatility than under steep volatility.

(4) The influence of the risk-free interest rate

For all of the initial credit ratings, the value of the credit-rating-linked coupon bond was higher when the risk-free interest rate was low. The difference between the initially set constant coupon and the credit-rating-linked coupon derived not from the risk-free interest rate itself, but rather from the credit spread. The riskfree interest rate only affected the value of the credit-rating-linked coupon bond through its impact on the discount rate of its cash flow.

\section{Summary and concluding remarks}

In this paper, we presented a structural valuation model for credit-rating-linked coupon bonds that incorporates the fact that an increased coupon payment resulting from a downgrade may deteriorate the potential of the issuing company to make future coupon and notional payments. Through numerical experiments, we demonstrated that our model reasonably captures this effect. A practical implication of our model is that the valuation of a credit-rating-linked coupon bond based on the JLT model tends to underestimate the value of the bond when its initial credit rating is high. However, the reverse is true when the initial credit rating is low.

\section{References}

[1] Bhanot K., Pricing Corporate Bonds with Rating-Based Covenants. The Journal of Fixed Income, March, pp. 57-64, 2003.

[2] Duffie, D. \& Singleton, K., Modeling Term Structures of Defaultable Bonds. Review of Financial Studies, 12, pp. 687-720, 1999.

[3] Jarrow, R.A. David L. \& Turnbull, S.M., A Markov Chain Model for the Term Structure of Credit Risk Spreads. Review of Financial Studies, 10(2), pp. 481-523, 1997.

[4] Jarrow, R. \& Turnbull S.M., Pricing Derivatives on Financial Securities Subject to Credit Risk. Journal of Finance, 50, pp. 53-85, 1995.

[5] Merton, R.C., On the Pricing of Corporate Debt: The Risk Structure of Interest Rates. Journal of Finance, 29, pp. 449-470, 1974. 